\title{
Enhancing microvasculature maps for Optical Coherence Tomography - Angiography (OCT-A)
}

\author{
M. Rapolu, P. Niedzwiedziuk, D. Borycki, P. Wnuk, and M. Wojtkowski \\ Institute of Physical Chemistry, Polish Academy of Sciences, ul. Kasprzaka 44/52, Warsaw, Poland.
}

Received September 05, 2018; accepted September 29, 2018; published September 30, 2018

\begin{abstract}
OCT-A is becoming more popular in recent years and there is a high demand to improve the quality of angiograms as well as to extract quantitative information. We applied various processing methods for microvasculature enhancement like Hessian-Frangi to a data set obtained with Bessel and Gaussian OCT systems. We used angiogenesis, fractal and multifractal analysis to extract more quantitative information. We applied the processing methods for healthy, stroke, tumor progression and the results of enhanced processing and quantitative analysis for those are presented in this letter.
\end{abstract}

Optical Coherence Tomography (OCT) is a non-invasive, and widely used 3D imaging modality used in the field of biomedical imaging. It offers superiority over the other imaging techniques in terms of high-resolution, fast image acquisition speed, higher penetration depth - all without the use of extraneous contrasting agents [1].

Recently there have been major advances in the field of OCT to achieve a high-quality contrast-enhanced signal and to visualize the vasculature at almost a double depth when compared to conventional OCT [2]. Some recent improvements include speckle-modulation OCT (SMOCT), which has demonstrated superiority by revealing anatomical features previously hidden by speckle noise [3]. Moreover, there have also been advancements in 4D live imaging using OCT [4]. The major disadvantage of OCT is that it is impossible to visualize microvasculature in real time from the raw OCT data. During recent years, several methods have been developed to visualize microvasculature. Post-processing methods involve different scanning protocols and algorithms to process raw interferogram data with their Fourier transform to obtain A-scans (line) which are concatenated, using different averaging techniques to achieve B-scans (crosssectional images). Later a phase analysis over time is used to achieve angiomaps and 4D OCT microvasculature, making the OCT technique even a more powerful tool. A high resolution and numerical aperture OCT system would do a better job imaging smaller vessels. It is also vital to improve the signal to noise ratio (SNR) in the OCT system, optimize the scan protocols and pattern (e.g. no. of oversampling), as well as raw data processing in order to obtain high-quality angiographic data. The correction of bulk axial motion of imaged samples can bring about a great improvement in contrast. The semiautomated approach will allow tracing blood vessels directly on the data and provide connectivity information more accurately [5]. We describe some issues, challenges and possible methods to analyze the OCT microvasculature quantitatively for a few datasets with healthy, stroke and cancer mice brain tissue.

The data were obtained with the Bessel beam OCM setup and Gaussian beam setup. The details of the Bessel beam set up was described by S. Tamborski et al. [6]. The standard Gaussian OCM setup was built using a Michelson interferometer. A short pulse laser $\sim 6 \mathrm{fs}$, central wavelength $800 \mathrm{~nm}$ was used with a focusing objective $4 \times$ lens with a numerical aperture of 0.13 and with a depth range of $\sim 300 \mu \mathrm{m}$ for a $3 \mathrm{~mm}$ collimated input beam. The bandwidth of the laser is $180 \mathrm{~nm}$ and the theoretical lateral resolution of the system is $\sim 2 \div 3 \mu \mathrm{m}$. The sensitivity of the system is calculated to be $92 \mathrm{~dB}$. The scanning protocol used for OCT-A consists of $400 \mathrm{~A}-$ scans and $400 \mathrm{~B}$-scans with 6 oversampling.

The laser beam is fed into a fiber and a 75/25 fiber coupler is used to split the beam into a reference and objective arm, respectively. The objective arm consists of an objective and the reflected beam from the sample is back coupled into the fiber using the same objective. After combining with the reference arm, interfering light is directed into a spectrometer.

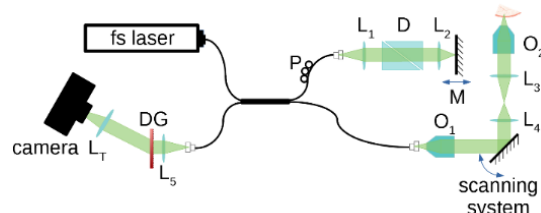

Fig. 1. Schematic diagram of the standard Gaussian OCM setup with a Michelson interferometer. $\mathrm{L}$ - lenses, $\mathrm{L}_{\mathrm{T}}$ - telecentric lens, DG - diffraction grating, O - objectives, M - mirror, D - dispersion compensation module, $\mathrm{P}$ - polarization controller, camera - Basler Sprint spl2048-140km

A Hessian-Frangi filter is a popular approach to enhance the vessels which combine the outputs of several Gabor filters at different scales and orientations. This method uses a ridge detector, enhancing tubular-like (e.g. vessels) geometrical structures. The eigenvalue decomposition extracts three orthonormal directions which are invariant up to a scaling factor when mapped by the Hessian matrix 
[7]. The major drawback of this method is that anything which is not narrow and elongated will also be attenuated in the output. A branch point where there is a major confluence of vessels sometimes does not look very ridge-like and can end up with a false disconnection at vessel junctions. To resolve this problem, we have upsampled our data before applying the Hessian-Frangi method. We can also alter filter independently for large vessels (using a larger sigma value), smaller vessels (using a smaller sigma value) or select the optimal sigma to get a more balanced rendering.

The major challenge in processing the OCT angiographic (OCT-A) data is the removal of artefacts related to the motion of the sample. One of the effective things we can do is to collect a few data sets and assume that these artifacts are dynamic components and microvasculature are static components. Later we can use a simple algorithm to eliminate these artifacts from microvasculature. This is the most efficient way to achieve high-quality angiographic data at the moderate cost of an increase in processing time and memory of the system. We also used a combination of median and Fast Fourier Transform (FFT) filters. A global thresholding would not be effective for the enhancement of small vessels. So, instead, we used an adaptive threshold method to enhance smaller microvasculature. The difference in an application with and without our adaptive thresholding can be clearly seen from Fig. 2(d) and Fig. 2(f), respectively.

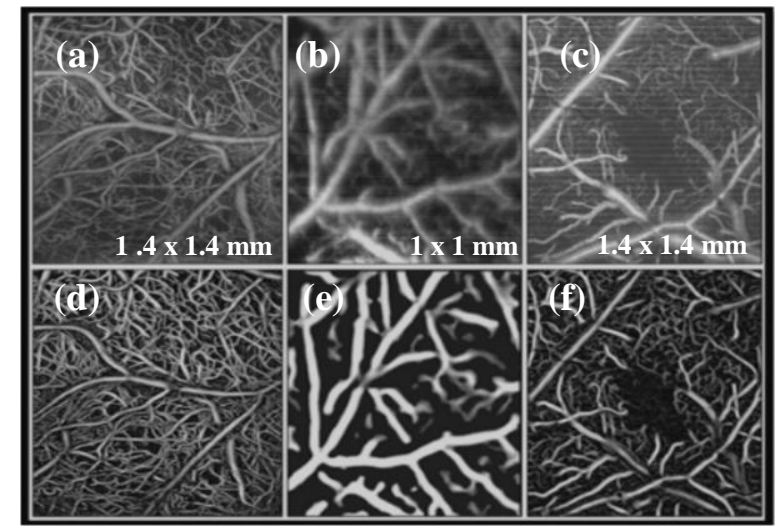

Fig. 2. Before (above) and after (below) application of Hessian-Frangi analysis and other post-processing methods for the improvement of visualization of cortical vessels applied to the dataset of (a), (d) healthy mouse, (b), (e) vessels system of mice brain region with induced stroke and (c), (f) mouse with the tumor. Fig. 2. (d), (f) are with and without the application of adaptive thresholding, respectively.

The increase in the visibility of vasculature with enhancement of smaller vessels and improvement in the quality of OCT-A data with almost zero background noise can be clearly seen in Fig. 2 (bottom row). This comparison suggests that the described processing steps are crucial to achieving the enhancement and high quality of microvasculature reconstructions.
Tumour can be often described as an uncontrolled and abnormal growth of tissue. Angiogenesis is a physiological process for de novo vascular network formation from pre-existing blood vessels [8]. During the growth process, the blood vessels undergo a variety of significant changes in size, vessel length, vessel diameter, junction density and structural, morphological changes from a few microns to hundreds of microns. The OCT system has the potential to evaluate very small tumors $(\sim 2$ microns), which is beneficial and greatly advantageous in understanding the repressed mechanism in their growth progression.

The advantage of using several evaluation points to measure physiological phenomena like vascular density, number and length of sprouts yields better understanding and more promising results while evaluating the tumor state. There are a variety of vascular metrics like fractal dimension, lacunarity, no. of endpoints, vessel area, average vessel length, no. of junctions, vessel junction density, vessel density developed in order to access the diagnosis and study the progression of a tumor [3]. There are only a few studies using OCT to evaluate these metrics for a tumor or stroke. This is because the contrast of OCT is not enough to image a tumor due to the change in the scattering properties of a tumor over time.

We have performed angiogenesis analysis by skeletonizing the OCT-A microvasculature data acquired for glioblastoma multiforme (GBM) tumor over a few days period.
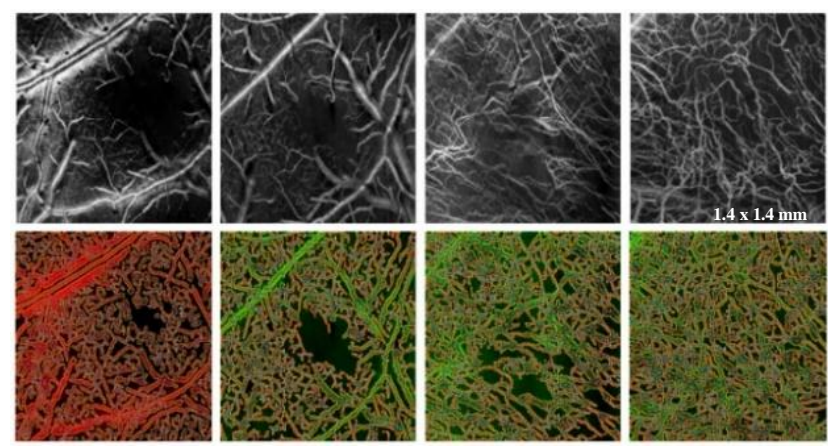

Fig. 3. Angiomaps (above) after background removal along with median and FFT filtering and their corresponding angiogenesis analysis on GBM tumor progression for a period of 1, 3, 8 and 14 days (left to right respectively).

A tumor is injected, imaged and analyzed in the cortical region of the mouse brain. Fig. 3 (top row) shows the microvasculature obtained from Gaussian beam OCT after applying median and FFT filtering for the GBM tumor progression for a period of $1,3,8$ and 14 days respectively (left to right). The bottom row in Fig. 3 represents their corresponding angiogenesis analysis. The angio parameters obtained are represented in Fig. 4 and Table 1. 
These parameters are strong indicators to prove that angiogenesis occurred during the tumor growth. The rate of growth can be quantified using these parameters. The increase in values of the vessel area, total number of junctions, junction density, average vessel length and lacunarity are strong indicators to demonstrate how rapid the progression of a tumor is.

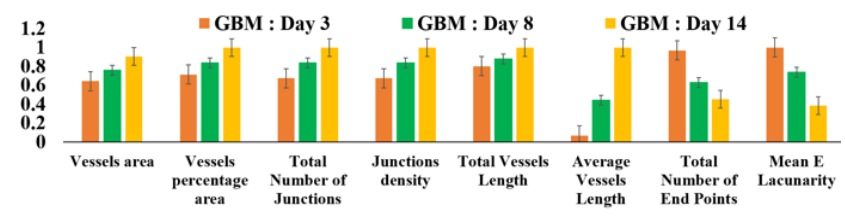

Fig. 4. Histogram of angiogenesis parameters for glioblastoma (GBM) progression dataset for $1,3,8$ and 14 days from left to right respectively.

Table. 1. Fractal dimension (FD), Lacunarity and density of vessels on glioblastoma (GBM) progression dataset obtained for different days

\begin{tabular}{|c|c|c|c|}
\hline $\begin{array}{c}\text { GBM } \\
\text { Progression }\end{array}$ & FD & Lacunarity & Density \\
\hline Day 3 & 1.8108 & 0.4843 & 0.2498 \\
\hline Day 8 & 1.8507 & 0.4757 & 0.3218 \\
\hline Day 14 & 1.8622 & 0.2938 & 0.3039 \\
\hline
\end{tabular}

The fractal analysis is done using a standard box-counting method with varying box sizes to estimate the fractal parameters. The details and the theory of multifractal and fractal analysis can be found in R. Lopes et al. [9]. The slope of the regression of $(\operatorname{lnN} / \ln \varepsilon)$ gives the Fractal dimension (FD) $(\mathrm{N}=$ number of foreground pixels and $\varepsilon$ $=$ box sizes in pixels dimension).
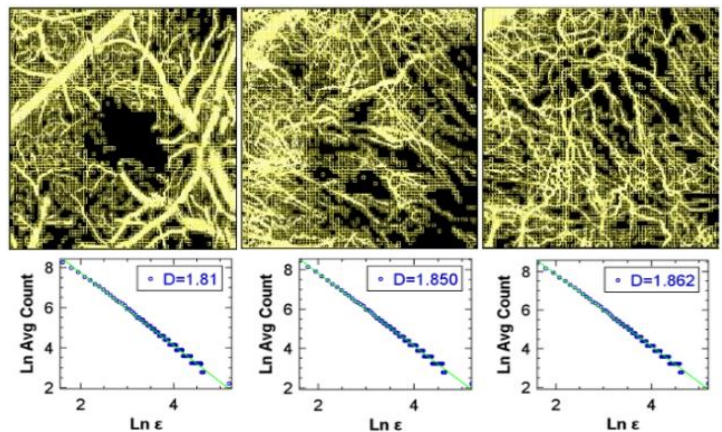

Fig. 5. Fractal analysis on glioblastoma (GBM) progression datase for 3,8 and 14 days from left to right respectively and their corresponding plots for box size vs. average count in log scale.

FD is useful in describing the pathological architecture of tumors and, perhaps more surprisingly, for yielding insights into the mechanisms of tumor growth and angiogenesis, complementing those obtained by modern molecular methods [10-11]. A fractal analysis was performed for the GBM dataset with a 10 grid position. Figure 5 (top row) shows the fractal analysis for one of the smallest box sizes for a period 3, 8 and 14 days (left to right) respectively. Their corresponding fractal dimension was plotted in the bottom row. An increase in the FD value indicates that the tumor has progressed and has become more tortuous.

In conclusions, we have demonstrated different methods to improve vasculature and to obtain high-quality microvasculature. These methods have shown the potential to quantify for different data sets like stroke and tumor. Such an approach would be very beneficial in our further studies by injecting intravenously Large Gold Nanorods (LGNRs) and visualizing a small tumor to increase contrast and the scattering signal from OCT. Temporal-microvasculature studies can be done with an optimized, high resolution and repetitive OCT system. We also plan to investigate the parameters introduced in this paper in more detail and later apply machine learning tools to quantify the data in the best possible way.

This work was funded by the National Science Center (NCN) 2016/22/A/ST2/00313. MW acknowledges funding from the European Union's Horizon 2020 research and innovation programme under grant agreement No 666295.

\section{References}

[1] J.G. Fujimoto, C. Pitris, S.A. Boppart, M.E. Brezinski, Neoplasia 2(12), 9 (2000).

[2] O. Liba, E.D. SoRelle, D. Sen, A. de la Zerda, Sci. Rep. 6(1), 23337 (2016). doi:10.1038/srep23337

[3] O Liba, M.D. Lew, E.D. SoRelle et al., Nat. Comm. 8, 15845 (2017). doi:10.1038/ncomms 15845

[4] K. Karnowski, A. Ajduk, B. Wieloch et al., Sci. Rep. 7(1), 4165 (2017). doi:10.1038/s41598-017-04220-8

[5] V.J. Srinivasan, S. Sakadžić, I. Gorczynska et al., Opt. Expr. 18(3), 2477 (2010). doi:10.1364/OE.18.002477

[6] S. Tamborski, H.C. Lyu, H. Dolezyczek et al., Biomed. Opt. Expr., 7(11), 4400 (2016). doi:10.1364/BOE.7.004400

[7] A.F. Frangi, W.J. Niessen, K.L. Vincken, M.A. Viergever, Lect. Notes Comput. Sc. 1496, 130 (1998).

[8] A.A. Ucuzian, A.A. Gassman, A.T. East, H.P. Greisler, J. Burn Care Res. 31(1), 158 (2010). doi: 10.1097/BCR.0b013e3181c7ed82

[9] R. Lopes, N. Betrouni Med. Image Anal. 13, 634 (2009).

[10] J.W. Baish, R.K. Jain, Cancer Res. 60(14), 3683 (2000). 